\title{
Determinantes do comportamento alimentar: uma revisão com enfoque na família
}

\author{
Determinants of eating behavior: a review \\ focusing on the family
}

Alessandra ROSSI

Emília Addison Machado MOREIRA²

Michelle Soares RAUEN ${ }^{3}$

\section{R E S U M O}

O comportamento alimentar ocupa atualmente um papel central na prevenção e no tratamento de doenças. A alimentação durante a infância, ao mesmo tempo em que é importante para o crescimento e desenvolvimento, pode também representar um dos principais fatores de prevenção de algumas doenças na fase adulta. Freqüentemente a família, os fatores sociais e os ambientais podem influenciar o padrão alimentar das crianças. O objetivo desta revisão foi abordar os diversos fatores ambientais envolvidos na aquisição de hábitos alimentares na infância. Os artigos selecionados foram pesquisados nas bases de dados Medline e SciELO considerando o período de 1978 a 2007. Foram utilizados os termos em ingles: children's eating patterns, children's dietary quality, determinants of children's eating patterns e em português. São apresentados os resultados de algumas investigações mais relevantes nesta área. Embora alguns desses fatores sejam inatos e, por isso, necessitem de um controle mais rigoroso, ressalta-se o papel determinante da família na formação dos hábitos alimentares saudáveis, pois a modificação de um hábito alimentar durante a fase adulta tem, em geral, alta taxa de insucesso. Além disso, outros fatores, como a escola, a rede social, as condições socioeconômicas e culturais, são potencialmente modificáveis e influenciam no processo de construção dos hábitos alimentares da criança e, conseqüentemente, do indivíduo adulto.

Termos de indexação: Comportamento alimentar. Educação alimentar e nutricional. Crianças. Hábitos alimentares.

\section{A B S T R A C T}

Eating behavior currently has a central role in the prevention and treatment of illnesses. Eating patterns during childhood, while important for growth and development, also represent one of the main factors that can

\footnotetext{
${ }_{1}^{1}$ Universidade Federal de Santa Catarina, Programa de Pós-Graduação em Nutrição. Florianópolis, SC, Brasil.

2 Universidade Federal de Santa Catarina, Centro de Ciência da Saúde, Departamento de Nutrição. Campus Universitário Trindade, 88040-970, Florianópolis, SC, Brasil. Correspondência para/Correspondence to: E.A.M. MOREIRA. E-mail: <addison@ccs.ufsc.br>.

3 Universidade Federal de Santa Catarina, Programa de Pós-Graduação em Odontologia. Florianópolis, SC, Brasil.
} 
prevent diseases in adulthood. Such eating patterns are frequently influenced by family, social and environmental factors. The objective of this review was to approach the many environmental factors involved in the acquisition of eating habits during childhood. The selected articles dating from 1978 to 2007 were found in the Medline and SciELO databases. The following keywords were used in Portuguese and English: children's eating patterns, children's dietary quality and determinants of children's eating patterns. The results of some more relevant researches in this area are presented. Although some factors are innate and thus need a more rigorous control, the determinant role played by the family in forming healthy eating habits is emphasized since attempts to modify eating habits during adulthood usually fail. Additionally, other factors such as school, social network and cultural and socioeconomic conditions are potentially modifiable and influence the process of building the child's eating habits and so that of the adult.

Indexing terms: Feeding behavior. Food and nutrition education. Children. Food habits.

\section{N T R O D U Ç Ã O}

Os principais problemas envolvendo a nutrição e a alimentação decorrem do excesso ou da carência de determinados nutrientes. Segundo estudiosos ${ }^{1}$, os problemas relacionados ao excesso de nutrientes, como à obesidade ou a carência destes, como a anemia, devem configurar entre as prioridades das ações atuais em saúde. Os sinais e os sintomas dessas doenças ocorrem após um período constante de inadequação no consumo alimentar. Dessa forma, o comportamento alimentar ocupa, atualmente, um papel central na prevenção e no tratamento de doenças.

Nesse contexto, a alimentação durante a infância, ao mesmo tempo em que é importante para o crescimento e desenvolvimento, pode também representar um dos principais fatores de prevenção de algumas doenças na fase adulta.

Na promoção de uma alimentação saudável dois aspectos devem ser ressaltados: a mudança de um comportamento alimentar a longo prazo é um objetivo com elevadas taxas de insucesso, e os hábitos alimentares da idade adulta estão relacionados com os aprendidos na infância². Esses dois aspectos apontam para que a intervenção na promoção de comportamentos alimentares saudáveis deva incidir com maior ênfase nos primeiros anos da infância, para que os mesmos permaneçam ao longo da vida.

A disponibilidade e o acesso ao alimento em casa, as práticas alimentares e o preparo do alimento, influenciam o consumo alimentar da criança. A população infantil é, do ponto de vista psicológico, socioeconômico e cultural, influenciada pelo ambiente onde vive, que, na maioria das vezes, é constituído pelo ambiente familiar. Dessa forma, as suas atitudes são, freqüentemente, reflexos desse ambiente. E quando o meio ambiente é desfavorável, o mesmo poderá propiciar condições que levem ao desenvolvimento de distúrbios alimentares que, uma vez instalados, poderão permanecer ao longo da vida ${ }^{3}$.

Uma vez que a alimentação pode exercer influência nos processos de saúde e doença no contexto familiar, o conhecimento acerca do comportamento alimentar das crianças é de grande relevância. Dessa forma, o objetivo deste artigo é rever dados publicados que abordem os fatores determinantes do comportamento alimentar de crianças, tendo como enfoque a unidade familiar.

\section{MÉ TOD OS}

Os artigos selecionados na presente revisão foram pesquisados nas bases de dados Medline e SciELO, considerando o período de 1978 a 2007. Foram utilizados os termos: padrões alimentares de crianças, qualidade alimentar da criança, determinantes dos padrões alimentares das crianças; e children's eating patterns, children's dietary quality, determinants of children's eating patterns. Na análise das publicações agruparam-se as informações de modo a caracterizar a relação entre a disponibilidade, a aceitabilidade e as preferências alimentares; a estrutura das refeições no contexto social, o ato de comer e determinantes cognitivos; a influência parental sobre a alimentação e 
a participação dos meios de comunicação, em especial a televisão no comportamento alimentar. Destaca-se, ainda, a reduzida freqüência de publicações nacionais referentes ao papel da família na determinação do comportamento alimentar.

\section{Disponibilidade, aceitabilidade e preferência alimentar}

O conhecimento de diferentes alimentos é essencial para a aquisição de uma alimentação variada, o que constitui uma das premissas fundamentais para uma alimentação saudável. A oferta de vegetais (verduras, folhosas e legumes) e frutas é determinante não só do consumo, mas também da preferência por este tipo de alimento ${ }^{4}$. Novas evidências têm indicado que a preferência pelo sabor-nutriente pode ser aprendida antes do desmame, e influenciar a escolha dos alimentos durante o desmame. O sabor do alimento afeta não somente a escolha, como também a quantidade de consumo alimentar ${ }^{5}$. Estudo de revisão, realizado por Wardle ${ }^{6}$, abordou aspectos sobre a relação da característica dos alimentos e o comportamento alimentar, enfatizando que as preferências alimentares mudam em conseqüência de experiências e aprendizado. O gosto dos alimentos pode ser associado a situações boas ou não. Esta é, provavelmente, a base do "efeito de familariedade", sendo esta mais evidente nas crianças. Wardle ${ }^{6}$ aborda ainda que existem evidências de que uma exposição sistemática (incluindo o "sabor" transmitido pelo leite materno) pode aumentar a familariedade com os alimentos, sendo isto verdadeiro para quem não gosta de vegetais.

Epstein et al. ${ }^{7}$, estudando 27 crianças com idades entre 6 e 11 anos e 27 pais, constataram que a redução na ingestão alimentar de gordura e açúcar pelas crianças pode ser obtida por mudanças dos hábitos alimentares dos pais. Além disso, a melhoria do hábito alimentar dos pais pode estender-se a todos os outros membros da família.
Dessa forma, a família fornece amplo campo de aprendizagem à criança. Os pais e outros membros familiares estabelecem um ambiente partilhado em que o convívio pode ser propício à alimentação excessiva e/ou a um estilo de vida sedentário. Pais que comem demais, muito rapidamente ou ignoram os sinais internos de saciedade oferecem um pobre exemplo aos seus filhos ${ }^{8}$. Por outro lado, os pais podem promover opções alimentares nutritivas às suas crianças, por meio de seleções alimentares sadias e de uma dieta saudável. As mães que selecionam preferencialmente os alimentos baseadas em critérios de qualidade e não apenas no sabor, oferecem refeições mais saudáveis às suas crianças ${ }^{9}$. Uma pesquisa com uma amostra de 225 crianças e 88 pais mostrou que o consumo de frutas, sucos naturais e vegetais é influenciado positivamente pela disponibilidade e pela acessibilidade desses alimentos em casa. Da mesma forma, os pais podem limitar os tipos e as quantidades de alimentos que contenham alto valor energético (por exemplo, bolacha recheada, refrigerante, snacks $)^{10}$.

Em geral as crianças escolhem os alimentos que lhes são servidos freqüentemente, e elas tendem a preferir os alimentos que facilmente estão disponíveis em casa ${ }^{11}$. A familiaridade com o alimento não é uma das suas características, mas resulta das experiências da criança com o mesmo. As evidências indicam que crianças tendem a preferir alimentos que Ihes são familiares, em detrimento dos que lhes são estranhos. Assim, as crianças tendem a consumir e preferir os alimentos aos quais são rotineiramente expostas ${ }^{12,13}$. Em um estudo de revisão ${ }^{14}$ foi verificado que tanto a disponibilidade como a acessibilidade é importante na escolha alimentar. Isto é, quando os alimentos estão acessíveis e prontos para o consumo, as crianças apresentam uma maior probabilidade de os comer. Como exemplo, Birch \& Fisher ${ }^{15}$ encontraram que, entre pré-escolares, a ingestão de frutas e verduras é mais elevada quando os alimentos estão disponíveis em locais acessíveis e em porções prontas para o consumo (isto é, salada de frutas, cenouras cortadas em palito). Embora as crianças não sejam particu- 
larmente aptas para pegar uma cenoura do refrigerador e as limpar, é mais provável que comam a cenoura que encontram limpa e cortada em tamanhos apropriados.

As crianças não comem aquilo de que elas não gostam ${ }^{16}$. Elas exercem um controle sobre a sua ingestão alimentar recusando ou comendo um alimento específico. Se a criança rejeita um alimento e por isso limita a ingestão alimentar, os pais ficam preocupados, pois, sabem que, nesse período de desenvolvimento, elas não devem omitir refeições. Freqüentemente a solução seria oferecer alimentos alternativos, porém nem sempre apresentam opções alimentares nutritivas. Esse tipo de problema pode conduzir a restrições alimentares, como, por exemplo, aos vegetais; e, conseqüentemente, a criança pode não receber uma alimentação balanceada, o que implicaria em prejuízo à sua saúde futura ${ }^{17}$. As crianças estão atentas aos alimentos de que gostam e àqueles que recusam, exercendo poder sobre seus pais durante as refeições. Birch, em uma série de estudos $11,17,18$, consistentemente tem mostrado que a aceitação a novos alimentos aumenta por meio de repetidas exposições, podendo requerer entre 8 a 10 exposições em crianças com 2 anos e de 8 a 15 vezes $^{11}$ para crianças entre 4 e 5 anos de idade, antes que se consigam modificações definitivas na alimentação ${ }^{17}$.

\section{A estrutura das refeições: o contexto social, 0 ato de comer e determinantes cognitivos}

As refeições em família representam um importante evento na promoção de uma alimentação saudável. Os alimentos servidos e as refeições oferecidas, em geral, são determinados pela família, ou seja, se a mesma se alimenta em casa ou se faz as refeições fora do lar. Uma pesquisa com uma amostra de 427 crianças entre dois e cinco anos de idade mostrou que quando os pais realizam a refeição com os filhos, cria-se uma atmosfera positiva, os pais servem como um modelo para o comportamento alimentar e as crianças tendem a melhorar a qualidade da alimentação ${ }^{19}$.

Vários estudos examinaram a contribuição do desjejum para a qualidade nutricional da alimentação em crianças ${ }^{20,21}$. Em geral, as crianças que realizaram desjejum apresentaram maior adequação em relação aos micronutrientes e um consumo de alimentos variados, comparado às crianças que não o realizam ${ }^{22,23}$. O desjejum pode ajudar no controle do peso corpóreo ${ }^{24}$, por meio da redução do consumo de gorduras e minimizando o impulso a comidas menos nutritivas ${ }^{25,26}$. O aumento da freqüência do jantar em família, em 16202 crianças entre 9 e 14 anos, foi associado a um padrão dietético saudável26. Swarr \& Richards ${ }^{27}$, em um estudo com 240 meninas, relataram que passar mais tempo com os pais foi um dos fatores que resultaram em melhorias das atitudes alimentares. Além disso, em um estudo com 427 crianças verificou-se que aquelas que realizavam as refeições na companhia dos pais e irmãos tendem a consumir um maior número de porções dos grupos dos cereais, verduras e vegetais, leite e derivados e carnes $^{19}$. Da mesma forma, Neumark-Sztainer et al. ${ }^{28}$, avaliando 4726 escolares, encontraram que a freqüência de realizar refeições com a família esteve associada positivamente ao consumo de frutas, vegetais, grãos e laticínios.

Fazer escolhas alimentares é um processo complexo, dinâmico e multifacetado, embutido nos relacionamentos sociais e que tem conseqüências a curto e longo prazo para a saúde ${ }^{29}$. As escolhas dos alimentos são formadas pelos sistemas de socialização culturais, e limitadas pela contingência do ambiente físico e social ${ }^{30}$. As refeições representam um importante evento nas interações familiares, com estruturas temporais portadoras de significado.

Entre outros fatores que interferem na estrutura das refeições pode-se citar o contexto social. Uma experiência positiva durante uma refeição pode induzir a preferência da criança aos alimentos, ao passo que uma experiência não prazerosa pode interferir negativamente na 
escolha dos alimentos. Assim, os pais que abordam em família o conhecimento sobre nutrição, ou, mais especificamente, sobre o valor nutricional dos alimentos, têm crianças que apresentam um maior conhecimento referente à alimentação. As mães que, freqüentemente, discutem tópicos como: "quais alimentos você gostou", "quais alimentos são bons para o nosso consumo", "vamos experimentar um novo alimento" ou "quais alimentos devemos preparar para refeição", fomentam na criança o interesse pelo alimento aumentando, assim, a sua aceitação $0^{31,32}$. Do mesmo modo, o efeito oposto sobre a aceitação de alimentos pode ser obtido quando os responsáveis forçam a criança a comer alimentos saudáveis, a fim de ganhar recompensas: "se você comer os seus vegetais você poderá comer pudim" 33 . Embora os pais utilizem esta abordagem para encorajar os filhos ao consumo de vegetais, esta tática tende a reduzir o gosto da criança pelo alimento e, inclusive, pode reforçar a preferência por pudim. A curto prazo, essas estratégias podem induzir as crianças a comer mais vegetais, no entanto, pesquisas sugerem que a longo prazo as tentativas de controle parental podem representar efeitos negativos na qualidade das dietas das crianças, inclusive, reduzindo sua preferência pelo alimento 34 .

As escolhas alimentares não dependem somente da disponibilidade e das preferências, mas também dos fatores cognitivos, tais como o conhecimento sobre os benefícios e o custo associados aos alimentos. Com esta percepção, O'Dea ${ }^{35}$ avaliou uma amostra de 213 crianças e adolescentes entre 7 e 17 anos com crenças relativas aos benefícios e às barreiras de uma alimentação saudável, e aos processos que consideravam eficazes para ultrapassar essas barreiras. As vantagens mais referidas em ter uma alimentação saudável foram: os benefícios psicológicos (aumento da auto-estima) e as sensações físicas agradáveis e, conseqüentemente, uma melhora no desempenho físico. As barreiras a uma alimentação saudável relatadas foram: conveniência no acesso e acessibilidade a alimentos não saudáveis, a pressão dos pares, o controle parental na alimentação (os alimentos disponíveis em casa não são saudáveis), as preferências e os impulsos alimentares não saudáveis no controle de estados emocionais. As estratégias mais referidas para lidar com as barreiras identificadas foram: o apoio parental, o planejamento (levar comida saudável ou não levar dinheiro para a escola), as estratégias cognitivas (lembrar-se dos inconvenientes da comida não saudável) e as estratégias educacionais (mais informação, publicidade aos alimentos saudáveis).

\section{Influência parental sobre a alimentação}

$\mathrm{O}$ aspecto de maior influencia ambiental para a criança é a família ${ }^{36,37}$. Em adolescentes americanos Branen \& Fletcher ${ }^{38}$ constataram que a influência parental foi associada ao desenvolvimento de práticas alimentares duradouras entre seus filhos. Por exemplo: os hábitos alimentares de adultos jovens, como comer todos os alimentos presentes no prato, usar o alimento como um incentivo ou uma ameaça, comer a sobremesa e realizar as refeições em horários programados, foram relacionadas com as mesmas práticas de alimentação utilizadas pelos pais deles durante a infância. Quando adultos jovens, ao selecionar um alimento eles relembram as considerações a respeito de nutrição ditadas por seus pais. Esta é uma constatação intrigante de como os pais podem influenciar, a longo prazo, as práticas alimentares de seus filhos ${ }^{38}$.

A influência parental sobre as escolhas alimentares pode se manifestar de várias maneiras: por meio da aquisição de gêneros alimentícios (ditados, em parte, pela renda, instrução e pela classe social), da religião e da cultura; seus comportamentos durante as refeições e suas reações aos alimentos podem servir de modelo para as crianças ${ }^{39}$; e na transmissão de informações sobre os alimentos (por exemplo, ensinando quais alimentos são saudáveis) ${ }^{36}$.

Para promover mudanças duráveis na alimentação das crianças e, conseqüentemente, 
possíveis melhorias para a saúde, deve-se compreender de que maneira as escolhas alimentares da família determinam a ingestão alimentar da criança e como estas escolhas alimentares se desenvolvem até a formação de um hábito alimentar $^{40}$.

As crianças aprendem a respeito do alimento não somente por suas experiências, mas também observando outros. As pesquisas ${ }^{41-46}$ realizadas por diferentes autores têm evidenciado similaridades com relação às preferências, à ingestão, à aceitação e à disposição para experimentar novos alimentos entre pais e filhos. Skinner et al. ${ }^{41}$ encontraram uma forte correlação entre as preferências alimentares de 117 pré-escolares, seus 213 pais e 47 irmãos mais velhos. Entretanto, nenhum membro da família pareceu ter maior influência sobre as preferências alimentares das crianças. Do mesmo modo, Burt \& Hertzler ${ }^{42}$ verificaram que os pais apresentaram uma influência positiva e igual em relação às preferências alimentares de suas crianças. Outros estudos demonstraram uma correlação positiva entre preferências de pais e suas crianças, mas as associações nem sempre eram significativas e variavam de acordo com a idade dos filhos. Uma meta-análise ${ }^{43}$ demonstrou uma correlação pequena, mas significativa entre as preferências dos pais e de suas crianças. As semelhanças entre as preferências foram similares entre os pais. Oliveira et al. ${ }^{44}$ relataram uma correlação entre a ingestão de nutrientes de 87 mães e 91 filhas, sugerindo que os pais sejam os objetivos de intervenções na tentativa de melhorar a alimentação das crianças. Pliner ${ }^{45}$ notou que as semelhanças entre as preferências alimentares de pais e filhos foram maiores do que aquelas relatadas por crianças e adultos e que a similaridade dos sexos entre pais e filhos aumentou as preferências. Logue et al. ${ }^{46}$, em um estudo com 77 crianças e suas mães, descreveu forte correlação nas preferências alimentares entre mulheres (mães-filhas).

Em uma revisão, Birch ${ }^{47}$ revela que não há nenhum dado que suporte ou refute a idéia de que as preferências alimentares e a dieta padrão formada na infância persistem durante a idade adulta. De fato, há, comparativamente, poucas evidências de que os hábitos alimentares persistem, mas isto se deve, principalmente, ao fato de existirem poucos estudos longitudinais. O estudo de coorte denominado Minnesota Heart Health Program, tinha como objetivo avaliar o comportamento alimentar de crianças. A pesquisa foi realizada anualmente, solicitando às crianças que selecionassem entre oito pares de alimentos, aqueles que habitualmente comeriam se pudessem escolhê-los. As crianças foram classificadas em uma dentre cinco categorias baseada no número de escolhas saudáveis realizadas no primeiro ano do estudo, e acompanhou-se o número médio de escolhas saudáveis dentro de cada grupo em cada ano subseqüente. Os resultados indicaram um aumento nas escolhas saudáveis com o passar dos anos, entretanto, não ocorreu nenhuma mudança gradual no ranking dos cinco grupos. Assim, as crianças que fizeram poucas escolhas saudáveis em 1983, também a fizeram em 1989, e aquelas que fizeram mais escolhas saudáveis continuaram a fazê-las. O acompanhamento paralelo dos cinco grupos sugeriu que nenhum foi especialmente susceptível a outros efeitos ambientais e biológicos, e que os hábitos alimentares persistem ${ }^{48}$.

Uma outra situação que tem sido estudada trata da relação da prática alimentar dos pais e do ganho de peso dos filhos. Wardle \& Carmell ${ }^{49}$, em estudo de revisão, compararam estudos realizados com crianças, mas com diferentes metodologias (4 estudos de casos-controle com uma amostragem de 18, 347, 634 e 214 crianças; um estudo transversal com uma amostra de 439 participantes, e estudos longitudinais de gêmeos com idades entre 4 a 7 anos de idade com um número de 3175 pares) com objetivo de identificar se o tipo de estudo alteraria as conclusões. Porém os revisores concluíram que independente do tipo de estudo, o impacto do estilo da alimentação sobre o ganho de peso das crianças é ainda incerto. Algumas evidências levam a crer que a falta de controle dos pais pode levar ao excessivo ganho de peso, enquanto outras sugerem que a 
imposição de certo estilo alimentar pode interferir no crescimento e no desenvolvimento da criança.

\section{Meios de comunicação: televisão}

Alguns trabalhos focalizam a relevância da televisão no comportamento alimentar de crianças. Um estudo realizado com 91 crianças e 91 pais demonstrou que as crianças cujas famílias realizam as refeições assistindo televisão apresentaram um menor consumo de frutas e verduras e um maior consumo de pizzas, salgadinhos e refrigerantes, comparativamente àqueles que não o fazem ${ }^{50}$. Ademais, uma pesquisa demonstrou que assistir à televisão durante as refeições está associado a um maior risco para deficiências nutricionais, em 3534 indivíduos com idade entre 2 e 24 anos $^{51}$. Assistir às propagandas comerciais dos alimentos induz a criança a pedir tais alimentos, e parece, assim, influenciar o seu padrão alimentar ${ }^{52}$. Os produtos alimentícios que são anunciados com maior freqüência tendem a apresentar um maior consumo, comparados àqueles que são menos anunciados (tais como: frutas e verduras) ${ }^{53}$.

Estudo de revisão, realizado por Taras \& Gage $^{54}$, em trabalhos desenvolvidos nos Estados Unidos, constataram que as crianças assistiam entre 21 a 22 horas semanais de televisão, das quais, aproximadamente, 3 horas correspondiam às publicidades de alimentos. Em $91 \%$ dos casos esta publicidade se referia aos alimentos com alto teor de gordura, açúcar e sal ${ }^{54}$. Adicionalmente, estudos em crianças de $4^{a}$ a $5^{a}$ série do ensino fundamental demonstraram que, independentemente do sexo, da capacidade de leitura, de fatores étnicos, ocupação e nível educacional dos pais, a televisão correlaciona-se positivamente a conceitos errôneos sobre os alimentos, à nutrição e a maus hábitos alimentares ${ }^{55}$. Além disso, pesquisas indicam forte correlação entre a seleção de alimentos de crianças de 3 a 8 anos e a publicidade desses alimentos veiculados pela televisão. Uma pesquisa com crianças e adolescentes entre 8 e 17 anos demonstrou que $78 \%$ das compras de alimentos pela família são influenciadas pelas escolhas dos filhos ${ }^{56}$.

Coon \& Tucker ${ }^{57}$, em uma revisão, verificaram associação direta entre o tempo de assistir televisão e a maior ingestão de alimentos energéticos, de refrigerantes (veiculado pela própria mídia televisiva) e a reduzida ingestão de frutas e verduras. Proctor et al. ${ }^{58}$ estudaram longitudinalmente um grupo de 106 pré-escolares até o início da puberdade, e verificaram que, durante a infância, aqueles que assistiram mais à televisão ganharam mais massa gorda do que magra ao longo dos anos.

\section{O N C L U S Ã O}

Embora a família seja um importante determinante na formação dos hábitos alimentares não se pode deixar de mencionar que outros fatores que não foram abordados nesta revisão, como a escola, a rede social, as condições socioeconômicas e culturais, são potencialmente modificáveis e influenciam no processo de construção dos hábitos alimentares da criança e, conseqüentemente, do indivíduo adulto.

Este estudo sugere que os pais desempenham um papel crucial no que diz respeito à compra e ao preparo dos alimentos, além do controle da qualidade dos alimentos ingeridos, já que as preferências alimentares das crianças são influenciadas pelas escolhas e pelos hábitos alimentares dos pais.

Destaca-se, também, que os pais devem monitorar o tempo em que seus filhos passam assistindo à televisão, em vista da natureza sedentária da atividade e de sua associação com a obesidade.

Dessa forma, a orientação educacional deve ter como objetivos a transmissão de informação nutricional, a mudança de crenças relativas à alimentação e, principalmente, deverá ser direcionada à família. Isso porque os pais têm um papel relevante no processo da aprendizagem das preferências e dos hábitos alimentares, podendo 
estimular uma prática alimentar saudável para os seus filhos.

\section{A GRADECIMENTO}

Ao Conselho Nacional de Desenvolvimento Científico e Tecnológico (CNPq), pela bolsa de produtividade e pelo apoio ao projeto de pesquisa (Processo n 401901/2005-0) do qual decorreu esta publicação.

\section{COLABORADORES}

A. ROSSI contribuiu com o levantamento bibliográfico e com a elaboração do artigo. E.A.M. MOREIRA colaborou com a elaboração do artigo e com a revisão crítica. M.S. RAUEN contribuiu com a elaboração do artigo.

\section{RE FER Ê N CIAS}

1. Frutuoso MFL, Vigantzky VA, Gambardella AMD. Níveis séricos de hemoglobina em adolescents segundo estágio de maturação sexual. Rev Nutr. 2003; 16(2):155-62.

2. De Bourdeaudhuij I. Family foods rules and healthy eating in adolescents. J Health Psychol. 1997; 2(1): 45-56.

3. Oliveira AM, Cerqueira EMM, Souza JS, Oliveira AC. Sobrepeso e obesidade infantil: influência de fatores biológicos e ambientais em Feira de Santana, BA. Arq Bras Endocrinol Metabol. 2003; 47(2):144-50.

4. Hearn MD, Baranowski T, Baranowski J, Doyle C, Lin LS, Smith M, et al. Environmental influences on dietary behavior among children: availability and accessibility of fruits and vegetables enables consumption. J Health Educ. 1998; 26(1):26-32.

5. Myers KP, Sclafani A. Development of learned flavor preferences. Dev Psychobiol. 2006; 48(5):380-8.

6. Wardle J. Eating behaviour and obesity. Obes Rev. 2007; 8(Suppl 1):73-5.

7. Epstein LH, Gordi CC, Raynor HO, Beddome M, Kilanowski CK, Paluch R. Increasing fruit and vegetable intake and decreasing fat and sugar intake in families at risk for childhood obesity. Obes Res. 2001; 9(3):171-8.

8. Lake AA. Longitudinal dietary changes from adolescence from adulthood: perceptions, attributions and evidence. Appetite. 2004; 42(3): 255-63.

9. Anliker JA, Laus MJ, Samonds KW, Beal V. Mothers' reports of their three-year-old children's control over foods and involvement in food-related activities. J Nutr Edu. 1992; 24(7):285-91

10. Cullen KW, Baranowski T, Owens E, Marsh T, Rittenberry L, Moor C. Availability, accessibility, and preferences for fruit, $100 \%$ fruit juice, and vegetables influence children's dietary behaviour. Health Educ Behav. 2003; 30(5):615-26.

11. Birch LL, Marlin DW. I don't like it; I never tried it: effects of exposure on two-year-old children's food preference. Appetite. 1982; 3(4):353-60.

12. Birch LL. Children's preferences for high-fat foods. Nutr Rev. 1992; 50(9):249-55.

13. Pliner $P$. The effects of mere exposure on liking for edible substance. Appetite. 1982; 3(3):283-90.

14. Baranowski T, Cullen KW, Baranowski J. Psychosocial correlates of dietary intake: advancing dietary intervention. Annu Rev Nutr. 1999; 19: 17-40.

15. Birch LL, Fisher JO. Development of eating behavior among children and adolescents. Pediatrics 1998; 101(3):539-49.

16. Brown R, Ogden J. Children's eating attitudes and behavior: a study of modeling and control theories of parental influence. Health Educ Res. 2004; 19(3):261-71.

17. Sullivan SA, Birch LL. Infant dietary experience and acceptance of solid foods. Pediatrics. 1994; 93(2): 271-7.

18. Sullivan SA, Birch LL. Pass the sugar, pass the salt: experiences dictates preference. Dev Psychol. 1990; 26(4):546-51.

19. Stanek K, Abbott D, Cramer S. Diet quality and the eating environment. J Am Diet Assoc. 1990; 90(11): 1582-4

20. Nicklas TA, O'Neil CE, Berenson GS. Nutrient contribution of breakfast, secular trends, and the role of ready-to-eat cereals: a review of data from the Bogalusa Heart Study. Am J Clin Nutr. 1998; 67(4):757S-63S

21. Siega-Riz AM, Popkin BM, Carson T. Trends in breakfast consumption for children in the United States from 1965-1991. Am J Clin Nutr. 1998; 67(4):748S-56S.

22. Bowman SA, Lino M, Gerrior SA, Basiotis PR. The healthy eating index: 1994-96. Washington (DC): U.S. Department of Agriculture, Center for Nutrition Policy and Promotion; 1998.

23. Ortega RM, Redondo MR, Lopez-Sobaler AM, Quintas ME, Zamora MI, Andres $\mathrm{P}$, et al. 
Associations between obesity, breakfast-time food habits and intake of energy and nutrients in a group of elderly Madrid residents. J Am Coll Nutr. 1996; 15(1):65-72.

24. Schlundt DG, Hill JO, Sbrocco T, Pope Cadle I, Sharp $\mathrm{T}$. The role of breakfast in the treatment of obesity: a randomized clinical trial. Am J Clin Nutr. 1992; 55(3):645-51.

25. Chao ESM, Vanderkooy PS. An overview of breakfast nutrition. J Can Diet Assoc. 1989; 50(4): 225-8.

26. Gillman MW, Rifas-Shiman $S L$, Frazier $A L$, Rockett $H R$, Camargo Jr CA, Field AE, et al. Family dinner and diet quality among older children and adolescents. Arch Fam Med. 2000; 9(3):235-40.

27. Swarr AE, Richards $\mathrm{MH}$. Longitudinal effects of adolescent's girl's pubertal development, perceptions of pubertal timing and parental relations on eating problems. Dev Psychol. 1996; 32(4):636-46.

28. Neumark-Sztainer D, Hannan Pj, Story M, Croll J, Perry C. Family meal patterns: associations with sociodemographic characteristics and improved dietary intake among adolescents. J Am Diet Assoc. 2003; 103(3):317-22.

29. Connors M, Bisogni C, Sobal J, Divine C. Manning values in personal food systems. Appetite. 2001; 36(3):189-200.

30. Murcott A. The Nation's diet: the social science of food choice. London: Longman; 1998.

31. Anliker JA, Laus MJ, Samonds KW, Beal VA. Parental messages and the nutrition awareness of preschool children. J Nutr Educ. 1990; 22(1):24-9.

32. Gillespie AH, Achterberg CL. Comparison of family interaction patterns related to food and nutrition. J Am Diet Assoc. 1989; 89(4):509-12.

33. Taylor $L$, Gallagher M, McCullough FSW. The role of parental influence and additional factors in the determination of food choices for preschool children. Int J Consum Stud. 2004; 28(4): 337-46.

34. Birch LL. Development of food preferences. Annu Rev Nutr. 1999; 19:41-62.

35. O'Dea JA. Why do kids eat healthful food? Perceived benefits of and barriers to healthful eating and physical activity among children and adolescents. J Am Diet Assoc. 2003; 103(4): 497-501.

36. Wardle J. Parental influences on children's diets. Proc Nutr Soc. 1995; 54(3):747-58.

37. Crockett JS, Sims LS. Environmental influences on children's eating. Soc Nutr Educ. 1995; 27(5):235-49.
38. Branen L, Fletcher J. Comparison of college students' current eating habits and recollections of their childhood food practices. J Nutr Educ. 1999; 31(6):304-10.

39. Ritchey N, Olson C. Relationships between family variables and children's preference for and consumption of sweets foods. Ecol Food Nutr. 1983; 13:257-66.

40. Longbottom PJ, Wrieden WL, Pine CM. Is there a relationship between the food intakes of Scottish $51 / 281 / 2$-year-olds and those of their mothers? J Hum Nutr Diet. 2002; 15(4):271-9.

41. Skinner J, Carruth B, Moran J, Houch K, Schmidhammer J, Reed A, et al. Toddler's food preferences: concordance with family member's preferences. J Nutr Educ. 1998; 30(1):17-22.

42. Burt J, Hertzler A. Parental influences on the child's preference. J Nutr Educ. 1978; 10(3):127-8.

43. Borah-Giddens J, Falciglia G. A meta-analysis of the relationship in food preferences between parents and children. J Nutr Educ. 1993; 25(2): 102-7.

44. Oliveira SA, Ellison RC, Moore LL, Gillman MW, Garrahie EJ, Singer MR. Parent child relationships in nutrient intake: the Framingham Children's Study. Am J Clin Nutr. 1992; 56(3):593-8.

45. Pliner P. Family resemblance in food preferences. J Nutr Educ.1983; 15(3):137-40.

46. Logue AW, Logue CM, Uzzo RG, McCarty MJ, Smith ME. Food preferences in families. Appetite. 1988; 10(3):169-80.

47. Birch LL. Psychological influences childhood diet. J Nutr. 1998; 128(2):407S-10S.

48. Kelder SH, Perry CL, Klepp KI, Lytle LL. Longitudinal tacking of adolescents smoking, physical activity, and food choice behaviors. Am J Public Health. 1994; 84(7):1121-6.

49. Wardle J, Carnell S. Parental feeding practices and children's weight. Acta Paediatr Suppl. 2007; 96(454):5-11.

50. Coon KA, Goldberg J, Rogers BL, Tucker KL. Relationships between use of television during meals and children's food consumption patterns. Pediatrics. 2001; 107(1):E7.

51. Serra-Majem L, Ribas L, Perez-Rodrigo C, GarciaClosas R, Pena-Quintana L, Aranceta J. Determinants of nutrient intake among children and adolescents: results from the enKid Study. Ann Nutr Metab. 2002; 46(Suppl 1):S31-S8.

52. Francis LA, Lee $Y$, Birch LL. Parental weight status and girls' television viewing, snacking, and body mass indexes. Obes Res. 2003; 11(1):143-51. 
53. Gallo EA. Food advertisements in the United States. In: America's eating habits: changes and consequences. Washington (DC): United States Department of Agriculture; 1998. Economics Research Service: Report AIB-750: 773-80.

54. Taras H, Gage M. Advertised foods on children's television. Arch Pediatr Adolesc Méd. 1995; 149(6): 649-52.

55. Signorielli N, Lears M. Television and children's conceptions of nutrition: unhealthy messages. Health Commun. 1992; 4(4):245-57.

56. Koplan JP, Liverman CT, KraaK VI. Preventing childhood obesity: health in the balance.
Washington: National Academy of Sciences; 2005.

57. Coon KA, Tucker KL. Television and children's consumption patterns. A review of the literature. Minerva Pediatr. 2002; 54(5):423-36.

58. Proctor MH, Moore LL, Gao D, Cupples LA, Bradlee ML, Hood MY, et al. Television viewing and changes in body fat from preschool to early adolescence: the Framingham Children's Study. Int J Obes Relat Metab Disord. 2003; 27(7):827-33.

Recebido em: 24/10/2006

Versão final reapresentada em: 11/3/2008 Aprovado em: 25/9/2008 